\title{
FARLEY-BUNEMAN INSTABILITY IN THE SOLAR CHROMOSPHERE
}

\author{
G. Gogoberidze ${ }^{1,2}$, Y. VoItenKo ${ }^{1,3}$, S. Poedts ${ }^{1,4}$, And M. Goossens ${ }^{1}$ \\ ${ }^{1}$ CPA/K.U.Leuven, Celestijnenlaan 200B, 3001 Leuven, Belgium \\ ${ }^{2}$ Georgian National Astrophysical Observatory, Chavchavadze State University, Kazbegi Ave. 2a Tbilisi-0160, Georgia \\ ${ }^{3}$ Belgian Institute for Space Aeronomy, Ringlaan-3-Avenue Circulaire, 1180 Brussels, Belgium \\ ${ }^{4}$ Leuven Mathematical Modeling \& Computational Science Centre, Belgium \\ Received 2008 December 1; accepted 2009 October 12; published 2009 October 27
}

\begin{abstract}
The Farley-Buneman instability (FBI) is studied in the partially ionized plasma of the solar chromosphere taking into account the finite magnetization of the ions and Coulomb collisions. We obtain the threshold value for the relative velocity between ions and electrons necessary for the instability to develop. It is shown that Coulomb collisions play a destabilizing role in the sense that they enable the instability even in the regions where the ion magnetization is larger than unity. By applying these results to chromospheric conditions, we show that the FBI cannot be responsible for the quasi-steady heating of the solar chromosphere. However, we do not exclude the instability development locally in the presence of strong cross-field currents and/or strong small-scale magnetic fields. In such cases, FBI should produce locally small-scale, $\sim 0.1-3 \mathrm{~m}$, density irregularities in the solar chromosphere. These irregularities can cause scintillations of radio waves with similar wave lengths and provide a tool for remote chromospheric sensing.
\end{abstract}

Key words: Sun: atmospheric motions - Sun: chromosphere

\section{INTRODUCTION}

The mechanism of chromospheric heating is a major puzzle of solar physics since it was discovered that the temperature in the solar chromosphere is much higher than what can be expected for a plasma in radiative equilibrium. The first possible scenario for explaining of chromospheric heating was proposed by Biermann (1946) and Schwarzschild (1948), who suggested that the inner atmosphere of the Sun is heated by acoustic waves that are generated in the convective zone. Later, theoretical and numerical studies (Stein 1967; Carlsson $\&$ Stein 1992) have demonstrated that acoustic waves are, in fact, abundantly generated in the convective zone and that these waves can, in principle, be responsible for chromospheric heating. However, measurements of the acoustic flux at different chromospheric levels usually fail to find sufficient energy to heat the whole chromosphere (Fossum \& Carlsson 2005). Also, random Alfvén waves can heat upper chromosphere via ionneutral collisions and generate slow shocks, which can explain the formation of spicules (Erdelyi \& James 2004).

As an alternative explanation for the chromospheric heating, it has been suggested (Parker 1988; Sturrock 1999) that impulsive nano-flares, powered by magnetic reconnection events, could be responsible for chromospheric heating. Although the observations show numerous transient brightenings on the Sun, these are insufficiently frequent and insufficiently energetic to explain the persistent UV emission of the chromosphere (Aschwanden et al. 2000). During solar flares, the chromosphere can be strongly heated and ionized locally by precipitating electron beams and evaporate upward, producing observed polarized $\mathrm{H} \alpha$ emission via collisional interaction with neutral surrounding hydrogen (Fletcher \& Brown 1998).

Yet another possibility for chromospheric heating is the resistive dissipation of electric currents (Rabin \& Moore 1984; Goodman 2004). Recent analysis of three-dimensional vector currents and temperatures, deduced from spectro-polarimetric observations of a sunspot from photospheric to chromospheric levels, has shown that, while resistive dissipation of large-scale (200 km and greater) currents can contribute to heat the sunspot chromosphere, it is not the dominant factor (SocasNavarro 2005). Although the possibility of chromospheric heating by resistive dissipation of small-scale currents cannot be excluded. Recently, it has been suggested that the Farley-Buneman (Farley 1963; Buneman 1963) instability (FBI), driven by convective motions, can be responsible for chromospheric heating (Liperovsky et al. 2000; Fontenla 2005; Fontenla et al. 2008). The FBI is known to create plasma irregularities in the terrestrial ionospheric E-region, at heights where the electrons are strongly magnetized. The interplay of the Earth's electric and geomagnetic field produces currents which give rise to the FBI. Similarly, in those places where the electrons are strongly magnetized, the collisional drag of the ions by neutral flows can cause the development of a similar instability. Using the decrement of the FBI derived by Farley (1963) and assuming a negligible ion magnetization, Fontenla et al. (2008) concluded that the FBI should be present at least in the upper half of the chromosphere. Earlier, the analysis of Liperovsky et al. (2000) had indicated that the FBI might operate in the chromosphere at heights $h>1000 \mathrm{~km}$.

However, the studies of the FBI in the chromosphere conditions are incomplete and they do not take into account two effects which under chromospheric conditions are important as we will show below. Firstly, if the finite magnetization of ions is taken into account (in the absence of Coulomb collisions), the Hall current perturbations weaken the FBI, and the system becomes stable for any neutral flow velocity when the ion magnetization factor $\kappa$ exceeds unity (Fejer et al. 1984). Therefore, this instability cannot operate in the upper solar chromosphere, where $\kappa>1$. Second, contrary to the E-layer plasma in the Earth's atmosphere, and top of the solar photosphere (Petrović et al. 2007), the ionization degree in the solar chromosphere is quite high $\left(10^{-2}-10^{-4}\right)$ and, consequently, Coulomb collisions cannot be ignored as is usually done in the study of the E-layer plasma.

In this Letter, we study the FBI taking into account both the finite magnetization of ions and the Coulomb collisions. We 
shall show that, in contrast to the situation in the ionospheric E-regions, the relatively high degree of ionization in the solar chromosphere makes the Coulomb collisions important for the FBI development. As a result, the instability can occur even in plasmas with the ion magnetization $\kappa$ larger than unity. However, by applying our analytical results to the solar chromosphere, we show that even though the FBI can sporadically develop in the chromosphere, it cannot be the main source of chromospheric heating.

\section{FORMALISM}

We consider a partially ionized plasma consisting of electrons, one species of singly charged ions and neutral hydrogen. In the upper solar chromosphere, the positively charged particles are mainly protons, whereas at lower altitudes the positive charge is dominated by heavy ions. We therefore do not further specify the type of ions, so that our results are applicable to both the upper and the lower chromosphere.

The dynamics of electrons and ions in such plasmas is governed by the continuity and the Euler equations, viz.,

$$
\frac{\partial n_{\alpha}}{\partial t}+\nabla \cdot\left(n_{\alpha} \mathbf{V}_{\alpha}\right)=0
$$

and

$$
\begin{aligned}
m_{\alpha} \frac{d_{\alpha} \mathbf{V}_{\alpha}}{\partial t}= & q_{\alpha}\left(\mathbf{E}+\frac{\mathbf{V}_{\alpha} \times \mathbf{B}}{c}\right)-\frac{\nabla n_{\alpha} \mathcal{K} T_{\alpha}}{n_{\alpha}} \\
& -m_{e} v_{e p}\left(\mathbf{V}_{\alpha}-\mathbf{V}_{\alpha^{*}}\right)-m_{\bar{\alpha}} v_{\alpha n}\left(\mathbf{V}_{\alpha}-\mathbf{V}_{n}\right) .
\end{aligned}
$$

Here, $\alpha=e, i$ denotes electrons or ions, $\alpha^{*}$ denotes the charged species opposite to $\alpha, \bar{\alpha}$ stands for $e$ for the electron equation and for proton $(p)$ for the ion equation. Also, $n$ corresponds to neutrals, and $n_{\alpha}$ denotes the density, $\mathbf{V}_{\alpha}$ is the averaged drift velocity, $m_{\alpha}$ is the mass, $T_{\alpha}$ is the temperature, $q_{\alpha}$ is the charge, $v_{\alpha \beta}$ is the collision frequency, $c$ is the speed of light, $K$ is The Boltzmann constant, and $d_{\alpha} / d t$ denotes the convective derivative.

For the electron-ion and electron-neutron collision frequencies, we use the following expressions (Braginskii 1965):

$$
v_{e i}=\frac{4(2 \pi)^{1 / 2} e^{4} n_{e} \Lambda}{3 m_{e}^{1 / 2}(\mathcal{K} T)^{3 / 2}}
$$

and

$$
v_{e n}=\sigma_{e n} n_{n} \sqrt{\frac{\mathcal{K} T_{e}}{m_{e}}}, \quad v_{i n}=v_{p n}=\sigma_{i n} n_{n} \sqrt{\frac{\mathcal{K} T_{p}}{m_{p}}},
$$

where $\Lambda$ corresponds to the Coulomb logarithm and in the former equation we take into account the fact that, regardless the mass of the dominant ion species, the ion-neutral collision frequency in the solar chromosphere is proportional to the thermal velocity of the neutral (hydrogen) component. The electron-neutral and ion-neutral collision cross sections are $\sigma_{e n}=3.0 \times 10^{-15} \mathrm{~cm}^{2}$ (Bedersen \& Kieffer 1971) and $\sigma_{i n}=2.8 \times 10^{-14} \mathrm{~cm}^{2}$ (Krstic \& Schultz 1999), respectively.

We assume that the system is penetrated by a uniform magnetic field $B$ in the $z$ direction and that the neutrals have a background velocity $\mathbf{V}_{n} \perp$ B. Equations (2) then yield a stationary solution for the background ion drift velocity

$$
\mathbf{V}_{i}=\frac{\left(1+\kappa \kappa_{1} \psi N\right) \mathbf{V}_{n}+\kappa \mathbf{V}_{n} \times \hat{z}}{1+\kappa_{1}^{2}}
$$

and for the so-called current velocity, i.e., the relative velocity between the ions and the electrons,

$$
\mathbf{U}_{0}=\mathbf{V}_{i}-\mathbf{V}_{e}=\frac{\mathbf{V}_{n}+\kappa_{1} \mathbf{V}_{n} \times \hat{z}}{1+\kappa_{1}^{2}}
$$

Here, $\kappa=\omega_{c p} / v_{p n}$ is proton magnetization, $\omega_{c \alpha} \equiv e B / m_{\alpha} c$ is the cyclotron frequency, $\kappa_{1}=\kappa(1+\psi N), \psi=v_{e n} v_{i n} / \omega_{c p} \omega_{c e}$, and $N=v_{e p} / \nu_{e n}$ is the ratio of Coulomb and electron-neutral collision frequencies.

On this background, we study linear electrostatic perturbations in the plane perpendicular to the background magnetic field. In order to simplify analysis we admit standard assumptions for the study of the FBI in the Earth's E-layer plasma (Oppenheim et al. 1996; Schunk \& Nagy 2000). We assume quasi-neutrality $\left(n_{e} \approx n_{i}\right)$. Technically, this means that, instead of using Poisson's equation, we use $\nabla \cdot \mathbf{J}=0$, where $\mathbf{J}$ denotes the electric current density. In addition, because $n_{e}$ and $n_{i}$ are indistinguishable, we use only one continuity equation. Finally, we treat the electrons as massless because the FBI occurs on an ion-neutral collision timescale which, for typical chromospheric conditions, strongly exceeds both the electron cyclotron gyration and the electron plasma oscillation timescales.

After linearizing Equations (1)-(2) and performing a Fourier transform, after longish but straightforward algebraic manipulations we arrive at the following dispersion equation

$$
\begin{aligned}
\frac{\omega-\mathbf{k} \cdot \mathbf{U}_{0}}{\psi} & +\frac{\left(1-i \omega / v_{i n}^{\prime}\right)^{2}+\kappa^{2}}{1-i \omega / v_{i n}^{\prime}} \omega+N\left(1-i \omega / v_{i n}^{\prime}\right) \\
& \times \omega+i(1+N) \frac{c_{s}^{2} k^{2}}{v_{i n}^{\prime}}=0,
\end{aligned}
$$

where $c_{s} \equiv\left[K\left(T_{e}+T_{i}\right) / m_{i}\right]^{1 / 2}$ is the sound velocity and $v_{i n}^{\prime}=m_{p} v_{i n} / m_{i}$. In the derivation of the dispersion equation, we neglected all terms of the order of the small parameter $\psi \kappa^{2} \sim m_{e} v_{e n} / m_{p} v_{p n} \sim 2.6 \times 10^{-3}$.

In the limit of low-frequency and long-wavelength perturbations $\left(|\omega|,\left|\mathbf{k} \cdot \mathbf{U}_{0}\right| \ll v_{i n}^{\prime}\right)$, we obtain the oscillation frequency $\omega_{r}$ and the growth rate $\gamma$ of the Farley-Buneman type instability:

$$
\omega_{r}=\frac{\mathbf{k} \cdot \mathbf{U}_{0}}{1+\bar{\psi}}
$$

and

$$
\gamma=\frac{\bar{\psi}\left(\mathbf{k} \cdot \mathbf{U}_{0}\right)^{2}}{v_{i n}^{\prime}(1+\bar{\psi})}\left[\frac{1-\kappa^{2} /(1+N)}{(1+\bar{\psi})^{2}}-\frac{k^{2} c_{s}^{2}}{\left(\mathbf{k} \cdot U_{0}\right)^{2}}\right],
$$

where $\bar{\psi} \equiv \psi(1+N)$.

\section{DISCUSSION AND CONCLUSIONS}

The terms proportional to $N=v_{e p} / v_{e n}$ in Equations (7)-(8) describe the effect of the electron-ion (Coulomb) collisions on the FBI and represent the main analytical result of this letter. When the Coulomb collisions are neglected $(N=0)$, then Equations (7)-(8) reduce to the well known result of Fejer et al. (1984), which indicates that in plasmas with $\kappa>1$, the FBI cannot develop regardless of the neutral drag velocity. In contrast, if the Coulomb collisions are sufficiently frequent, the FBI can appear even when the ions are relatively highly magnetized. The dependence of $N$ on height, based on data of a 


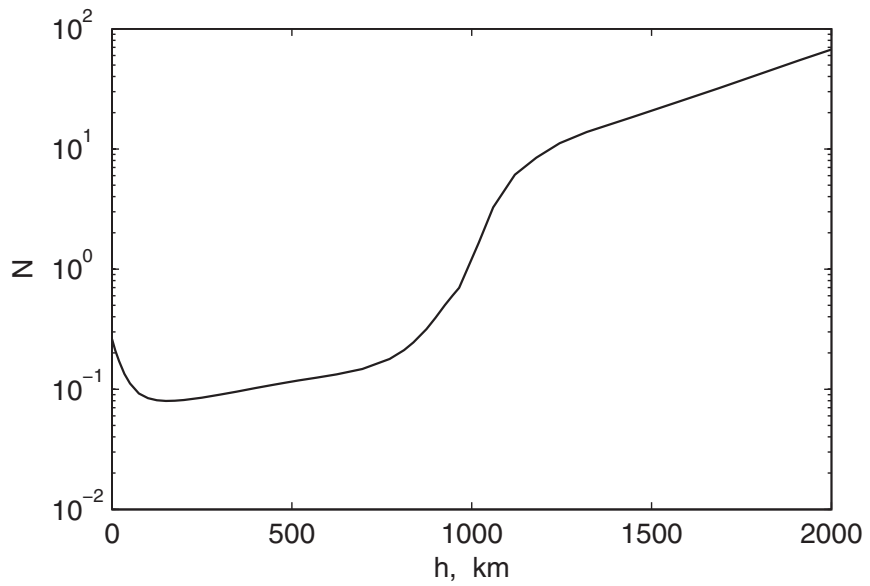

Figure 1. Ratio of Coulomb and electron-neutral collision rates $N$ as a function of height in the solar chromosphere.

semi empirical chromospheric model SRPM 306 (Fontenla et al. 2007), is shown in Figure 1. It is seen that the Coulomb collisions dominate the electron-neutral collisions $(N>1)$ in the upper half of the solar chromosphere, at heights $h>1000 \mathrm{~km}$. At these heights they cannot be ignored and facilitate the FBI.

From Equation (8), we determine the threshold value of the relative velocity $U_{0}^{\text {cr }}$ necessary to trigger the FBI in the framework of the model SRPM 306. As follows from Equation (8), a necessary condition for the FBI is that $U_{0}^{\text {cr }}$ exceeds $c_{s}$. Figure 2 shows the dependence of $U_{0}^{\text {cr }}$ on height with (solid lines) and without (dashed lines) Coulomb collisions, for two values of magnetic field: $B_{0}=30 \mathrm{G}$ (thin lines) and $B_{0}=60 \mathrm{G}$ (thick lines). The left panel corresponds to the protons and the right panel to the representative ions with $m_{i}=30 m_{p}$.

The results obtained here allow us to draw conclusions about the possible role of the FBI in the internetwork chromospheric heating. The threshold value of the current velocity necessary to trigger the FBI corresponds to the current density $J_{0}=e n_{0 e} U_{0}^{\text {cr }}$. Even in the lower chromosphere, where the positively charged particles are mainly heavy ions and $U_{0}^{\text {cr }} \sim 2 \mathrm{~km} \mathrm{sec}^{-1}$, very strong current densities $J_{0} \sim 2.4 \times 10^{6}$ statampere $\mathrm{cm}^{-2}$ are required for the FBI to develop. According to recent observations of Socas-Navarro (2005), at length scales of the order $200 \mathrm{~km}$ and higher, the typical values of the observed currents are much smaller, $\sim 5 \times 10^{4}$ statampere $\mathrm{cm}^{-2}$.

Recent high-resolution Hinode observations have revealed that at a spatial scale $\gtrsim 230 \mathrm{~km}$ the magnetic field $B$ in the internetwork photosphere cannot exceed few hundred $G$ with a mean value $\sim 100 \mathrm{G}$ (Orosco Suarez et al. 2007; Lites et al. 2008). Then, $B$ in the overlying lower chromosphere is expected to be smaller, at least at that spatial scales, so the bound $B \leqslant 100 \mathrm{G}$ that we used in the lower chromosphere seems to be reasonable.

As is usually assumed, the supercritical currents can be driven at smaller scales by neutral microturbulent motions (Fontenla et al. 2007), and consequently the FBI can develop and contribute to chromospheric heating at such length scales. The magnetic field $B$ generated by the supercritical current $J_{0}$ with the length scale $L$ is given by the Ampere law: $B \sim 4 \pi J_{0} L / c$. This relation provides a link between the characteristic magnetic field and characteristic length scale relevant to FBI. For instance, it shows that in the regions where $B<100 \mathrm{G}$, the supercritical currents can only appear at very small length scales $L<1 \mathrm{~km}$. In this context, we should also note that according to a semi empirical model by Sánchez Almeida and co-authors (Sánchez Almeida \& Lites 2000; Socas-Navarro \& Sánchez Almeida 2003 ) very strong $\left(B \sim 10^{2}-10^{3} \mathrm{G}\right)$ small-scale $(L \sim 1-10 \mathrm{~km})$ magnetic fields can exist in the internetwork photosphere. If such fields do exist, they can provide the field gradients and current densities in the overlaying chromosphere, which are sufficient for triggering the FBI. But as we show below, the dominant role of the FBI in the chromospheric heating can be excluded also at that small length scales. Based on the energetic arguments it can be shown that the FBI driven by such smallscale currents produces a negligible heating in comparison to the heating produced by the frictional dissipation of the relative ion-neutral motion.

The rate of frictional dissipation in partially ionized plasmas is (Braginskii 1965)

$$
\begin{aligned}
Q_{f r}= & m_{e} n_{e} v_{e i}\left(\mathbf{V}_{e}-\mathbf{V}_{i}\right)^{2}+m_{e} n_{e} v_{e n}\left(\mathbf{V}_{e}-\mathbf{V}_{n}\right)^{2} \\
& +m_{p} n_{e} v_{i n}\left(\mathbf{V}_{p}-\mathbf{V}_{i}\right)^{2}
\end{aligned}
$$

where the terms on the right-hand side are due to electronion, electron-neutral and ion-neutral frictions, respectively. By substituting here Equations (4)-(5), we obtain

$$
Q_{f r}=m_{e} n_{e} e v_{e n} U_{0}^{2}\left(1+\kappa^{2}+N+\frac{1}{\psi}\right) .
$$

At relatively low heights in the solar chromosphere, where the positive charge is dominated by heavy ions, the friction dissipation is dominated by the ion-neutral friction (term
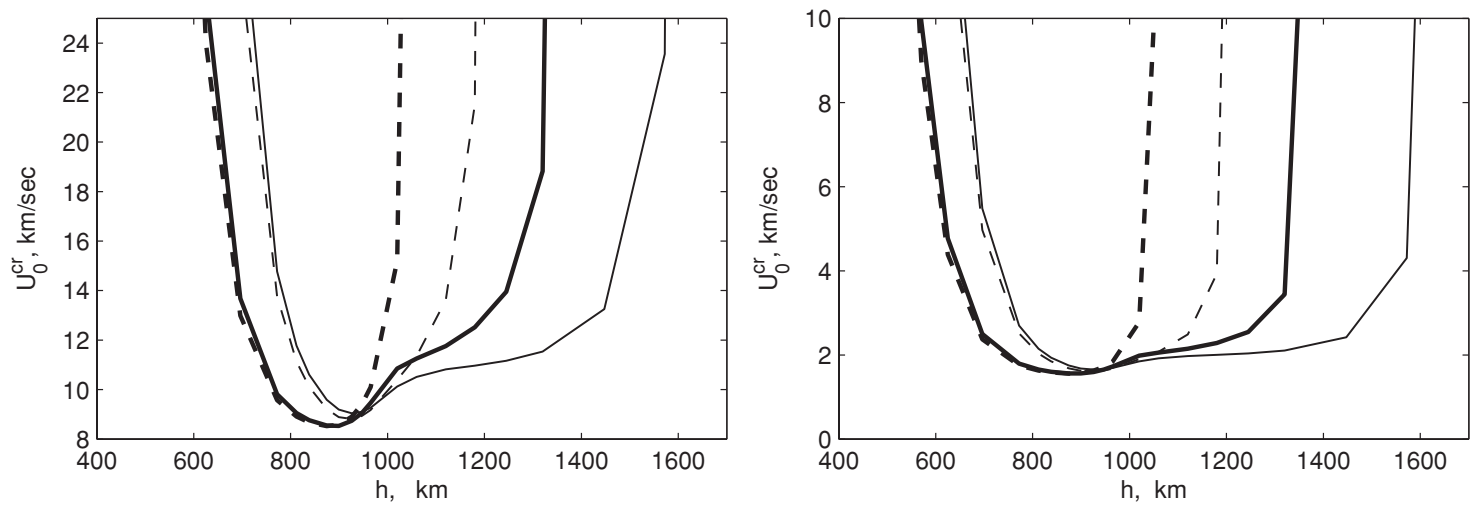

Figure 2. Dependence of the threshold value of the velocity $U_{0}^{\text {cr }}$ on height with (solid lines) and without (dashed lines) Coulomb collisions, for $B=30 \mathrm{G}$ (thin lines) and for $B=60 \mathrm{G}$ (thick lines). The left panel corresponds to the protons and the right panel to ions with $m_{\mathrm{i}}=30 m_{\mathrm{p}}$. 
proportional to $1 / \psi)$. On the other hand, the heating rate by FB waves cannot exceed the energy pumping rate produced by the FBI, which can be estimated as the (double) growth rate times the kinetic energy of the FB perturbations, $d W_{\mathrm{FB}} /(d t) \sim$ $2 \gamma W_{\mathrm{FB}}$. This estimation follows from $v_{i} \sim \exp (\gamma t)$ and hence $W_{\mathrm{FB}} \sim\left(v_{i}\right)^{2} \sim \exp (2 \gamma t)$, where $v_{i}$ is the ion velocity perturbation due to FBI. Taking this as an upper limit for the possible heating rate by the FBI, we obtain $Q_{\mathrm{FBI}} \sim 2 \gamma W_{\mathrm{FB}}^{\prime} \sim$ $n m_{\mathrm{i}}\left(v_{\mathrm{i}}^{\dagger}\right)^{2} \gamma$, where primed quantities correspond to the level of instability saturation. According to Oppenheim \& Otani (2006) the nonlinear saturation of the FBI occurs at $n^{\prime} / n \sim 10^{-2}-10^{-1}$. Noting that the perturbed ion velocity is related to the density perturbation $n^{\prime} / n$ as $v_{i}^{\prime} / u_{T i} \sim\left(k u_{T i} / v_{i n}^{\prime}\right) n^{\prime} / n$, where $u_{T i}$ is the ion thermal velocity, and estimating from Equation (8) $\gamma \sim \bar{\psi} /(1+\bar{\psi})\left(k U_{0}^{\text {cr }}\right)^{2} / \nu_{i n}^{\prime}$ in the lower chromosphere, we obtain the ratio of heating rates

$$
\begin{aligned}
\frac{Q_{\mathrm{FBI}}}{Q_{f r}} \sim & \left(\frac{k U_{0}^{\mathrm{cr}}}{v_{i n}^{\prime}}\right)^{2}\left(\frac{k u_{T i}}{v_{i n}^{\prime}}\right)^{2}\left(\frac{u_{T i}}{U_{0}^{\mathrm{cr}}}\right)^{2}\left(\frac{n^{\prime}}{n}\right)^{2} \\
& \times\left(\sqrt{\frac{m_{p}}{m_{\mathrm{e}}}} \frac{\sigma_{i n}}{\sigma_{e n}}\right) \frac{\bar{\psi}^{2}}{(1+\bar{\psi})^{2}} .
\end{aligned}
$$

Analysis of the full dispersion Equation (6) shows that the growth rate reaches its maximum for $k U_{0}^{\text {cr }} / v_{i n}^{\prime} \sim 0.2-0.3$. Then, by setting $u_{T i} \sim U_{0}^{\text {cr }} \sim c_{S}$ and $n^{\prime} / n \sim 0.05$, we obtain $Q_{\mathrm{FBI}} / Q_{f r} \sim 4 \times 10^{-3} \bar{\psi}^{2} /(1+\bar{\psi})^{2}<4 \times 10^{-3}$. Consequently, the frictional heating is several order larger than the heating by FBI. Note that the last multiplier in Equation (11) makes the ratio of the heating rates even smaller in the upper chromosphere, where $\bar{\psi} \ll 1$. Furthermore, if strong currents at unresolved scales necessary to trigger the FBI would be presented in the chromosphere with significant filling factor, then the frictional dissipation would produce much more heat than it is necessary to compensate the radiative losses of the chromosphere. Indeed, taking the heights around $H \sim 850 \mathrm{~km}, U_{0}^{\mathrm{cr}} \sim 2 \mathrm{~km} \mathrm{~s}^{-1}$, $n_{n} \sim 10^{14} \mathrm{~cm}^{-3}, n \sim 10^{11} \mathrm{~cm}^{-3}, \bar{\psi} \sim 1$, Equation (10) yields $Q_{f r} \sim 10 \mathrm{erg} \mathrm{cm}^{-3} \mathrm{~s}^{-1}$, which is more than one order larger than the characteristic chromospheric heating rate $\sim 0.2 \mathrm{erg} \mathrm{cm}^{-3} \mathrm{~s}^{-1}$.

The remaining possibility is that the strong overthreshold currents are built up sporadically by the neutral turbulence at small length scales, $10 \mathrm{~m} \leqslant L \leqslant 1 \mathrm{~km}$, and drive sporadic FBI events in such small-scale areas unresolvable for modern observations. We note that the small-scale plasma irregularities produced by the FBI can scatter radio waves, and hence provide a diagnostic tool for strong cross-field chromospheric currents if they exist. This last issue requires further investigation. In particular, our preliminary analysis of the full dispersion Equation (6) has already demonstrated that for typical chromospheric parameters the strongest FBI occurs at a characteristic wavelength that varies with height in the range $0.1-3 \mathrm{~m}$. In the middle chromosphere at $1000 \mathrm{~km}$, the maximum instability growth rate $\gamma \approx 2 \times 10^{3} \mathrm{~s}^{-1}$ occurs for waves with characteristic wavelengths $\lambda \approx 16 \mathrm{~cm}$. The decimetric radio emission should effectively interact with the electron density irregularities produced by the FBI, which should lead to observable scintillations of the decimetric radio emission. Our model becomes inapplicable for the current length scales approaching the FBI wavelengths, $L \sim L_{\mathrm{FBI}}=0.1-1 \mathrm{~m}$, but such smallscale supercritical currents seem to be unrealistic in the solar chromosphere.
In our analysis, we assumed the background electric field to be zero. In general, the FBI is driven by the cross field relative motion of charged plasma species, which can be caused also by the background electric field if it exists. However, to be efficient in driving the FBI, the electric field should be of the order $E \sim B c_{s} / c \sim 10^{-3}$ statvolt $\mathrm{cm}^{-1}$, which is about 2 orders of magnitude larger than the strongest electric fields expected in the chromosphere (Goodman 2000).

In summary, we determined the threshold current velocity for the FBI to occur in a partially ionized plasma taking into account the finite ion magnetization and the electron-ion Coulomb collisions. We have shown that in the presence of Coulomb collisions, the FBI can occur even when the ion magnetization is larger than unity. Applying these analytical results to the solar chromosphere, we concluded that the FBI cannot be the main mechanism for the chromospheric heating at any length scales. The FBI at small length scales cannot be excluded, but the heating produced by the FBI cannot compete with the frictional heating under chromospheric conditions. We suggest that the small-scale irregularities generated by the FBI can be used for remote diagnostics of strong cross-field currents in the solar chromosphere.

The authors are grateful to Juan Fontenla for providing SRPM 306 data and to Jovo Vranjes for helpful discussions. We thank the referee for his constructive comments that significantly improved the Letter. G.G. acknowledges partial support from Georgian NSF grants ST06/4-096 and ST07/4-193. Y.V. acknowledges partial support by STCE (Solar-Terrestrial Center of Excellence) under the project "Fundamental science." These results were obtained in the framework of the projects GOA/2009009 (K.U. Leuven), G.0304.07 (FWO-Vlaanderen) and C 90347 (ESA Prodex 9). Financial support by the European Commission through the SOLAIRE Network (MTRN-CT-2006-035484) is gratefully acknowledged.

\section{REFERENCES}

Aschwanden, M. J., Tarbell, T. D., Nightingale, R. W., Schrijver, C. J., Title, A., Kankelborg, C. C., Martens, P., \& Warren, H. P. 2000, ApJ, 535, 1047

Bedersen, B., \& Kieffer, L. J. 1971, Rev. Mod. Phys., 43, 601

Biermann, L. 1946, Naturwissenschaften, 33, 118

Braginskii, S. I. 1965, Reviews of Plasma Physics (New York: Consultants Bureau), 205

Buneman, O. 1963, Phys. Rev. Lett., 10, 285

Carlsson, M., \& Stein, R. 1992, ApJ, 397, L59

Erdélyi, R., \& James, S. P. 2004, A\&A, 427, 1055

Farley, D. T. 1963, J. Geophys. Res., 68, 6083

Fejer, B. G., Providakes, J., \& Farley, D. T. 1984, J. Geophys. Res., 89, 7487

Fletcher, L., \& Brown, J. C. 1998, A\&A, 338, 737

Fontenla, J. M. 2005, A\&A, 442, 1099

Fontenla, J. M., Balasubramaniam, K. S., \& Harder, J. 2007, ApJ, 667, 1243

Fontenla, J. M., Peterson, W. K., \& Harder, J. 2008, A\&A, 480, 839

Fossum, A., \& Carlsson, M. 2005, Nature, 435, 919

Goodman, M. L. 2000, ApJ, 533, 501

Goodman, M. L. 2004, A\&A, 424, 691

Krstic, P. S., \& Schultz, D. R. 1999, J. Phys. B: At. Mol. Opt. Phys., 32, 3485

Liperovsky, V. A., Meister, C.-V., Liperovskaya, E. V., Popov, K. V., \& Senchenkov, S. A. 2000, Astron. Nachr., 321, 129

Lites, B. W., et al. 2008, ApJ, 672, 1237

Oppenheim, M., \& Otani, N. 2006, J. Geophys. Res., A111, 03302

Oppenheim, M., Otani, N., \& Ronchi, C. 1996, J. Geophys. Res., A 101, 17273

Orozco Suárez, D., et al. 2007, ApJ, 670, L61

Parker, E. N. 1988, ApJ, 330, 474 
Petrović, D., Vranjes, J., \& Poedts, S. 2007, A\&A, 461, 277

Rabin, D., \& Moore, R. 1984, ApJ, 285, 359

Sánchez Almeida, J., \& Lites, B. W. 2000, ApJ, 532, 1215

Schunk, R. W., \& Nagy, A. F. 2000, Ionospheres (Cambridge: Cambridge Univ. Press)
Schwarzschild, M. 1948, ApJ, 107, 1

Socas-Navarro, H. 2005, ApJ, 633, L57

Socas-Navarro, H., \& Sánchez Almeida, J. 2003, ApJ, 593, 581

Stein, R. 1967, Sol. Phys., 2, 385

Sturrock, P. A. 1999, ApJ, 421, 45 